Bull. Austral. Math. Soc.

$49 F 20,53 C 40,58 \mathrm{E} 15$

VOL. 44 (1991) [325-336]

\title{
NONEXISTENCE OF STABLE CURRENTS IN SUBMANIFOLDS OF A PRODUCT OF TWO SPHERES
}

\author{
XUE-SHAN ZHANG
}

Dedicated to Professor Yuen-da Wang on his 68th birthday

\begin{abstract}
By using techniques of the calculus of variations in geometric measure theory, we investigate the nonexistence of stable integral currents in $S^{n_{1}} \times S^{n_{2}}$ and its immersed submanifolds. Several vanishing theorems concerning the homology group of these manifolds are established.
\end{abstract}

\section{INTRODUCTION}

For any compact Riemannian manifold $M$, a theorem of Federer and Fleming [2] tells us that any non-trivial integral homology class in $H_{p}(M, Z)$ corresponds to a stable integral current. By establishing a second variation formula for minimal integral currents and applying it to different situations of $M$, Lawson and Simons [3] investigated the nonexistence of stable integral currents in $M$ and showed some vanishing theorems concerning the $p$ th singular homology group $H_{p}(M, Z)$ of $M$ with integer coefficients. For an immersed submanifold $M$ of the unit sphere $S^{n}$, they showed the following theorem.

Theorem . (Lawson and Simons [3]). Let $M^{m}$ be a compact submanifold of $S^{n}$ with the second fundamental form $h$, and $p$ a given integer, $p \in(0, m)$. If for any $x \in M$ and any orthonormal basis $\left\{e_{i}, e_{\alpha}\right\}(i=1, \ldots, p ; \alpha=p+1, \ldots, m)$ of $T_{x} M$ the following condition is satisfied

$$
B(\xi)=\sum_{i, \alpha}\left[2\left\|h\left(e_{i}, e_{\alpha}\right)\right\|^{2}-\left\langle h\left(e_{i}, e_{i}\right), h\left(e_{\alpha}, e_{\alpha}\right)\right\rangle\right]<p(m-p),
$$

then there is no stable $p$-current in $M$ and hence

$$
H_{p}(M, Z)=H_{m-p}(M, Z)=0
$$

In this paper we shall extend the above theorem. We shall introduce a selfadjoint linear operator $Q^{A}$ on a p-subspace $V$ of the tangent space $T_{x} M$. Replacing $B(\xi)$ in (0.1) by the trace of $Q^{A}$, we shall prove the following two theorems.

Received 6 November 1990

Copyright Clearance Centre, Inc. Serial-fee code: 0004-9729/91 \$A2.00+0.00. 
THEOREM 1. There is no stable p-current in $S^{m_{1}} \times S^{m_{2}}$ and

$$
H_{p}\left(S^{m_{1}} \times S^{m_{2}}, Z\right)=0,
$$

where $0<p<m_{1}+m_{2}, p \neq m_{1}$ and $p \neq m_{2}$.

THEOREM 2. Let $\phi: M^{m} \rightarrow S^{n_{1}} \times S^{n_{2}}$ be an isometric immersion of a compact Riemannian manifold $M$ in $S^{n_{1}} \times S^{n_{2}}$, and $p$ a given integer, $p \in(0, m)$. If for any $x \in M$ and any $p$-subspace $V$ of $T_{x} M$

$$
\operatorname{tr} Q^{A}<0,
$$

then there is no stable p-current in $M$ and

$$
H_{p}(M, Z)=H_{m-p}(M, Z)=0 .
$$

\section{INTEgRal CURRENTS}

For later convenience, in this section we shall give a brief description of integral currents. We refer the reader to $[2,3]$ for more details.

Let $M^{m}$ be an $m$-dimensional compact Riemannian manifold with Riemannian metric $\langle$,$\rangle and Levi-Civita connection \nabla$. And let $\mathcal{H}^{p}$ denote Hausdorff $p$-measure on $M$. A subset $S$ of $M$ is called a $p$-rectifiable set if $S$ is a countable union of disjoint $p$-dimensional $C^{1}$ submanifolds, up to sets of $\mathcal{H}^{p}$-measure zero. Consider over $S$ an $\mathcal{H}^{p}$-measurable section $\xi: S \rightarrow \wedge^{p} T M$ with the property that for $\mathcal{H}^{p}$-almost all $x \in S$, $\xi_{x}$ is a simple vector of unit length which represents $T_{x} S$. Such a pair $(S, \xi)$ is called an oriented, $p$-rectifiable set.

The set of rectifiable $p$-currents is defined by

$$
\mathcal{R}_{p}(M)=\left\{\mathcal{S}=\sum_{n=1}^{\infty} n \mathcal{S}_{n} ; \mathcal{S}_{n}=\left(S_{n}, \xi_{n}\right), M(\mathcal{S})=\sum_{n=1}^{\infty} n \mathcal{H}^{p}\left(S_{n}\right)<\infty\right\}
$$

It can be thought of as the group of infinite, summable chains of oriented p-rectifiable sets.

For an oriented, $p$-rectifiable set $\mathcal{S}=(S, \xi)$ and a smooth $p$-form $\omega \in \wedge^{p}(M)$, define

$$
\mathcal{S}(\omega)=\int_{S} \omega\left(\xi_{x}\right) d S^{p}(x)
$$

This assigns to $\mathcal{S}$ a continuous linear functional on $\wedge^{P}(M)$. The boundary of $\mathcal{S}$ is defined as the linear functional on $\wedge^{p-1}(M)$ given by

$$
(\partial \mathcal{S})(\omega)=\mathcal{S}(d \omega)
$$


In the case that $\mathcal{S}$ and $\partial \mathcal{S}$ are both rectifiable currents, $\mathcal{S}$ is called an integral p-current. The space of integral p-currents is denoted by $\mathcal{T}_{p}(M)$. The direct sum $\mathcal{T}_{*}(M)=\bigoplus_{p} \mathcal{T}_{p}(M)$ together with $\partial: \mathcal{T}_{*}(M) \rightarrow \mathcal{T}_{*}(M)$ forms a differential chain complex. For this complex there is the following theorem.

ThEOREM. (Federer and Fleming [2]). For each $p \geqslant 0$ there is a natural isomorphism

$$
H_{p}\left(\mathcal{T}_{*}(M)\right) \cong H_{p}(M, Z)
$$

And for each $\alpha \in H_{p}\left(\mathcal{T}_{*}(M)\right)$ there exists a current $S \in \alpha$ of "least mass", that is,

$$
M(\mathcal{S}) \leqslant M\left(\mathcal{S}^{\prime}\right)
$$

for all $\mathcal{S}^{\prime} \in \alpha$.

Consider a current $\mathcal{S} \in \mathcal{R}_{p}(M)$ and a smooth vector field $X \in C(T M)$. Let $\phi_{t}: M \rightarrow M$ be the 1-parameter group of local diffeomorphisms generated by $X$. Then the rectifiable current $\phi_{t^{*}}(\mathcal{S})$ is given by

$$
\phi_{t^{*}}(\mathcal{S})(\omega)=\mathcal{S}\left(\phi_{t}^{*} \omega\right)
$$

Its "mass" is

$$
M\left(\phi_{t^{*}} \mathcal{S}\right)=\int_{M}\left\|\phi_{t^{*}} \overrightarrow{\mathcal{S}}\right\| d\|\mathcal{S}\|,
$$

where $\overrightarrow{\mathcal{S}}$ is the field of oriented tangent planes of $\mathcal{S}=\sum_{n} n \mathcal{S}_{n}$, for each $x \in S_{n}$, $\overrightarrow{\mathcal{S}}_{\boldsymbol{x}}=\xi_{n}(x)$.

A current $\mathcal{S} \in \mathcal{R}_{p}(M)$ is said to be stable if for each vector field $X$ there is an $\varepsilon>0$ such that

$$
M\left(\phi_{t^{*}} \mathcal{S}\right) \geqslant M(\mathcal{S})
$$

for $|t|<\varepsilon$. This implies that for each $X$ we have

$$
\left.\frac{d}{d t} M\left(\phi_{t^{*}} \mathcal{S}\right)\right|_{t=0}=0,\left.\quad \frac{d^{2}}{d t^{2}} M\left(\phi_{t^{*}} \mathcal{S}\right)\right|_{t=0} \geqslant 0
$$

The following variation formulas have been derived by Lawson and Simons [3].

$$
\begin{aligned}
\left.\frac{d}{d t} M\left(\phi_{t^{*}} \mathcal{S}\right)\right|_{t=0}= & \int\left\langle a^{X}(\overrightarrow{\mathcal{S}}), \overrightarrow{\mathcal{S}}\right\rangle d\|\mathcal{S}\| \\
\left.\frac{d^{2}}{d t^{2}} M\left(\phi_{t^{*}} \mathcal{S}\right)\right|_{t=0}= & \int\left\{-\left\langle a^{X}(\overrightarrow{\mathcal{S}}), \overrightarrow{\mathcal{S}}\right\rangle^{2}+\left\langle a^{x} a^{x}(\overrightarrow{\mathcal{S}}), \overrightarrow{\mathcal{S}}\right\rangle+\left\|a^{X}(\overrightarrow{\mathcal{S}})\right\|^{2}\right. \\
& \left.+\left\langle\nabla{ }_{x, \overrightarrow{\mathcal{S}}} X, \overrightarrow{\mathcal{S}}\right\rangle\right\} d\|\mathcal{S}\|,
\end{aligned}
$$


where $a^{X}: \wedge^{p} T_{x} M \rightarrow \wedge^{p} T_{z} M$ is a linear map given by

$$
\begin{gathered}
a^{X}\left(X_{1} \wedge \ldots \wedge X_{p}\right)=\sum_{j} X_{1} \wedge \ldots \wedge a^{X}\left(X_{j}\right) \wedge \ldots \wedge X_{p} \\
a^{X}\left(X_{j}\right)=\nabla_{X_{j}} X
\end{gathered}
$$

and $\nabla x, X: \wedge^{p} T_{x} M \rightarrow \wedge^{p} T_{x} M$ is another linear map defined by

$$
\begin{aligned}
\nabla_{X, X_{1} \wedge \ldots \wedge X_{p}} X & =\sum_{j} X_{1} \wedge \ldots \wedge\left(\nabla_{X, x_{j}} X\right) \wedge \ldots \wedge X_{p} \\
\nabla_{X, x_{j}} X & =\nabla_{X} \nabla_{X_{j}} X-\nabla_{\nabla_{X} X_{j}} X
\end{aligned}
$$

To any simple $p$-vector $\xi \in \wedge^{p} T_{x} M$ and $X \in C(T M)$, let $\phi_{t}$ be the flow generated by $X$, and define

$$
Q_{\xi}(X)=\left.\frac{d^{2}}{d t^{2}}\left\|\phi_{t^{*}} \xi\right\|\right|_{t=0}
$$

Then the expression (1.1) can be denoted by

$$
\left.\frac{d^{2}}{d t^{2}} M\left(\phi_{t^{*}} \mathcal{S}\right)\right|_{t=0}=\sum_{n} n \int_{S_{n}} Q_{\xi_{n}}(X) d \mathcal{H}^{P}(x)
$$

If $X=\nabla f$ for some $f \in C^{3}(M)$, from $[3, \mathrm{p} .436]$ we have

$$
Q_{\xi}(X)=\left[\sum_{j}\left\langle a^{X}\left(e_{j}\right), e_{j}\right\rangle\right]^{2}+2 \sum_{j, \alpha}\left\langle a^{X}\left(e_{j}\right), e_{\alpha}\right\rangle^{2}+\sum_{j}\left\langle\nabla_{X, e_{j}} X, e_{j}\right\rangle
$$

where $\left\{e_{i}, e_{\alpha}\right\}(i=1, \ldots, p ; \alpha=p+1, \ldots, m)$ is an orthonormal basis of $T_{x} M$ and $\xi=e_{1} \wedge \ldots \wedge e_{p}$.

\section{A SELFADJOINT LINEAR OPERATOR}

For a $p$-rectifiable set $S$ in $M$, we know that at $\mathcal{H}^{p}$-almost all points $x \in S$, there exists an approximate $p$-space $T_{x} S \subset T_{x} M$, to $S$. In this section we shall introduce a selfadjoint linear operator on $T_{x} S$. Its trace is equal to the trace of $Q_{\xi}$ given by (1.3).

Let $\phi: M^{m} \rightarrow N^{n}$ be an isometric immersion of a Riemannian manifold $M$ into a Riemannian manifold $N$. The Levi-Civita connections of $M$ and $N$ are denoted by $\nabla$ and $\bar{\nabla}$ respectively. For any $X, Y \in C(T M)$, we have

$$
\bar{\nabla}_{X} Y=\nabla_{X} Y+h(X, Y)
$$


where $h$ is the second fundamental form of the immersion $\phi$. If $V(N, M)$ is the normal bundle of $M$ in $N$, for a smooth section $\nu \in C(V(N, M))$ we have

$$
\bar{\nabla}_{X} \nu=-A_{\nu} X+\bar{\nabla}_{X}^{\frac{1}{\nu}} \nu
$$

where $A_{\nu}$ is the so-called the shape operator determined by $\nu$. We know that

$$
\left\langle A_{\nu} X, Y\right\rangle=\langle h(X, Y), \nu\rangle .
$$

For a given integer $p \in(0, m)$ let $V$ be a $p$-dimensional subspace in $T_{x} M$. Define a map $B_{\nu}: V \rightarrow V$ associated with $A_{\nu}$ by

$$
B_{\nu} X=\text { orthogonal projection of } A_{\nu} X \text { onto } V,
$$

where $X \in V$. If $\left\{e_{i}\right\}$ is an orthonormal basis of $V$, we have

$$
B_{\nu} X=\sum_{i}\left\langle A_{\nu} X, e_{i}\right\rangle e_{i}
$$

It may be seen that $B_{\nu}$ is a selfadjoint linear operator on $V$ because $A_{\nu}$ is selfadjoint linear. Let $\left\{\nu_{\lambda}\right\}$ be an orthonormal basis of the normal space $V_{x}(N, M)$ and $A_{\lambda}=A_{\nu_{\lambda}}$. Define a selfadjoint linear map $Q^{A}: V \rightarrow V$ associated with the immersion $\phi$ by

$$
Q^{A} X=\sum_{\lambda}\left[2\left(\sum_{i}\left(A_{\lambda}^{2} X, e_{i}\right) e_{i}-B_{\lambda}^{2} X\right)-\left(\operatorname{tr} A_{\lambda}-\operatorname{tr} B_{\lambda}\right) B_{\lambda} X\right],
$$

where $X \in V$ and $\left\{e_{i}\right\}$ is an orthonormal basis of $V, Q^{A}$ is independent of the choice of orthonormal bases of $V_{x}(N, M)$ and $V$. And its trace is

$$
\begin{aligned}
\operatorname{tr} Q^{A} & =\sum_{i}\left\langle Q^{A} e_{i}, e_{i}\right\rangle \\
& =\sum_{\lambda}\left[2\left(\sum_{i}\left\langle A_{\lambda}^{2} e_{i}, e_{i}\right\rangle-\operatorname{tr} B_{\lambda}^{2}\right)-\left(\operatorname{tr} A_{\lambda}-\operatorname{tr} B_{\lambda}\right) \operatorname{tr} B_{\lambda}\right] .
\end{aligned}
$$

Let $\left\{e_{\alpha}\right\}$ be an orthonormal basis of $V^{\perp}$ which is the orthogonal complement of $V$ in $T_{x} M$. Then $\left\{e_{i}, e_{\alpha}\right\}$ is an orthonormal basis of $T_{x} M$ and

$$
\begin{aligned}
\sum_{i}\left(A_{\lambda}^{2} e_{i}, e_{i}\right\rangle & =\sum_{i, j}\left\langle A_{\lambda} e_{i}, e_{j}\right\rangle^{2}+\sum_{i, \alpha}\left\langle A_{\lambda} e_{i}, e_{\alpha}\right\rangle^{2}, \\
\operatorname{tr} B_{\lambda}^{2} & =\sum_{i}\left\langle B_{\lambda}^{2} e_{i}, e_{i}\right\rangle=\sum_{i, j}\left\langle A_{\lambda} e_{i}, e_{j}\right\rangle^{2} .
\end{aligned}
$$


Hence (2.4) becomes

$$
\operatorname{tr} Q^{A}=\sum_{\lambda}\left[2 \sum_{i, \alpha}\left\langle A_{\lambda} e_{i}, e_{\alpha}\right\rangle^{2}-\left(\operatorname{tr} A_{\lambda}-\operatorname{tr} B_{\lambda}\right) \operatorname{tr} B_{\lambda}\right] .
$$

Now assume that $\psi: N^{n} \rightarrow R^{l}$ is an isometric immersion of the Riemannian manifold $N$ in the Euclidean space $R^{l}$. Let $D$ be the Levi-Civita connection on $R^{l}$. Associated with the isometric immersion $x=\psi \circ \phi: M^{m} \rightarrow R^{l}$, the shape operator $A_{\nu}^{\prime}$ determined by $\nu \in C\left(V\left(R^{l}, M\right)\right)$ is given by

$$
A_{\nu}^{\prime} Y=-\left(D_{Y} \nu\right)^{T}
$$

where $Y \in C(T M)$. In particular, if $\nu \in C(V(N, M))$,

$$
\begin{aligned}
A_{\nu}^{\prime} Y & =-\left(D_{Y} \nu\right)^{T}=-\left[\bar{\nabla}_{Y} \nu+\bar{h}(\nu, T)\right]^{T} \\
& =-\left(-A_{\nu} Y+\nabla_{Y}^{\perp} \nu\right)^{T}=A_{\nu} Y
\end{aligned}
$$

and if $\nu \in C\left(V\left(R^{l}, N\right)\right)$,

$$
A_{\nu}^{\prime} Y=\left(\bar{A}_{\nu} Y\right)^{T}
$$

For a given vector $v \in R^{l}$, we define two vector fields $v^{T}$ and $v^{\perp}$ on $M$ by

$$
\begin{aligned}
& v^{T}(x)=\text { orthogonal projection of } v \text { onto } T_{x} M, \\
& v^{\perp}(x)=\text { orthogonal projection of } v \text { onto } V_{x}\left(R^{l}, M\right) .
\end{aligned}
$$

To any unit, simple $p$-vector $\xi \in \wedge^{P} T_{x} M$, we shall calculate the quadratic form $Q_{\xi}\left(v^{T}\right)$ given by (1.3). Using (2.6), we have

$$
\begin{gathered}
a^{v^{T}}(Y)=\nabla_{Y} v^{T}=\left(D_{Y} v-D_{Y} v^{\perp}\right)^{T}=A_{v \perp}^{\prime} Y, \\
\nabla_{Y}^{\perp} v^{\perp}=\left(D_{Y} v-D_{Y} v^{T}\right)^{\perp}=-h^{\prime}\left(v^{T}, Y\right) .
\end{gathered}
$$

These imply

$$
\nabla_{v} T, Y v^{T}=\left(\nabla_{v}^{*} A^{\prime}\right)_{v \perp} Y-A_{h^{\prime}\left({ }^{T} T, v^{T}\right)} Y
$$

where $\nabla_{v}^{*} A^{\prime}$ is the derivative with respect to the connection of Van der WaerdenBortolotti ([1, p.65]). Putting (2.10) and (2.11) into (1.3), we obtain

$$
\begin{aligned}
Q_{\xi}\left(V^{T}\right)= & {\left[\sum_{j}\left\langle A_{v \perp}^{\prime} e_{j}, e_{j}\right\rangle\right]^{2}+2 \sum_{j, \alpha}\left\langle A_{v \perp}^{\prime} e_{j}, e_{\alpha}\right)^{2} } \\
& +\sum_{j}\left\langle\left(\nabla_{v}^{*} T A^{\prime}\right)_{v \perp} e_{j}, e_{j}\right\rangle-\sum_{j}\left\langle A_{h^{\prime}\left(v^{\prime} T,{ }^{\prime} T\right.}^{\prime} e_{j}, e_{j}\right\rangle .
\end{aligned}
$$


Let $(S, \xi)$ be an oriented, $p$-rectifiable set. With a point $x \in S$ associate a tangent $p$-space $V=T_{x} S \subset T_{x} M$. Choose an orthonormal basis $\left\{e_{i}, e_{\alpha}\right\}$ of $T_{x} M$ such that $\left\{e_{i}\right\}$ is a basis of $V$ and $\xi=e_{1} \wedge \ldots \wedge e_{p}$. Suppose that $\left\{\nu_{\sigma}\right\}$ is an orthonormal basis of $V_{x}\left(R^{l}, M\right)$ associated with the immersion $\psi \circ \phi: M \rightarrow R^{l}, A_{\sigma}^{\prime}=A_{\nu_{\sigma}}^{\prime}$, and $Q^{A^{\prime}}$ is the selfadjoint linear operator on $V$ defined by (2.3). Consider $Q_{\xi}$ as a quadratic form defined on the set

$$
\theta=\left\{v^{T} \in C(T M) ; v \in R^{l}, v^{T}\right. \text { is defined by (2.9)\}. }
$$

There is the following relation between the quadratic form $Q_{\xi}$ and the operator $Q^{A^{\prime}}$.

Proposition 1. $\operatorname{tr} Q_{\xi}=\operatorname{tr} Q^{A^{\prime}}$, where

$$
\operatorname{tr} Q^{A^{\prime}}=\sum_{\sigma}\left[2 \sum_{i, \alpha}\left\langle A_{\sigma}^{\prime} e_{i}, e_{\alpha}\right\rangle^{2}-\left(\operatorname{tr} A_{\sigma}^{\prime}-\operatorname{tr} B_{\sigma}^{\prime}\right) \operatorname{tr} B_{\sigma}^{\prime}\right] .
$$

Proof: Observing that at the given point $x \in M,\left\{e_{i}, e_{\alpha}, \nu_{\sigma}\right\}$ is an orthonormal basis of $R^{l}$ and $\left(\nabla_{v^{T}}^{*} A^{\prime}\right)_{v \perp}=0$ as $v^{T}=0$ or $v^{\perp}=0$, from (2.12) we have

$$
\begin{aligned}
\operatorname{tr} Q_{\xi}= & \sum_{i} Q_{\xi}\left(e_{i}\right)+\sum_{\alpha} Q_{\xi}\left(e_{\alpha}\right)+\sum_{\sigma} Q_{\xi}\left(\nu_{\sigma}\right) \\
= & -\sum_{i, j}\left\langle A_{h^{\prime}\left(e_{i}, e_{i}\right)}^{\prime} e_{j}, e_{j}\right\rangle-\sum_{\alpha, j}\left\langle A_{h^{\prime}\left(e_{\alpha}, e_{\alpha}\right)}^{\prime} e_{j}, e_{j}\right\rangle \\
& +\sum_{\sigma}\left\{\left[\sum_{j}\left\langle A_{\sigma}^{\prime} e_{j}, e_{j}\right\rangle\right]^{2}+2 \sum_{j, \alpha}\left\langle A_{\sigma}^{\prime} e_{j}, e_{\alpha}\right\rangle^{2}\right\} \\
= & -\sum_{\sigma}\left[\sum_{i, j}\left\langle A_{\sigma}^{\prime} e_{i}, e_{i}\right\rangle\left\langle A_{\sigma}^{\prime} e_{j}, e_{j}\right\rangle+\sum_{\alpha, i}\left\langle A_{\sigma}^{\prime} e_{\alpha}, e_{\alpha}\right\rangle\left\langle A_{\sigma}^{\prime} e_{i}, e_{i}\right\rangle\right] \\
& +\sum_{\sigma}\left\{\left[\sum_{j}\left\langle A_{\sigma}^{\prime} e_{j}, e_{j}\right\rangle\right]^{2}+2 \sum_{j, \alpha}\left\langle A_{\sigma}^{\prime} e_{j}, e_{\alpha}\right\rangle^{2}\right\} \\
= & \sum_{\sigma, i, \alpha}\left[2\left\langle A_{\sigma}^{\prime} e_{i}, e_{\alpha}\right\rangle^{2}-\left\langle A_{\sigma}^{\prime} e_{\alpha}, e_{\alpha}\right\rangle\left\langle A_{\sigma}^{\prime} e_{i}, e_{i}\right\rangle\right] .
\end{aligned}
$$

Since

and

$$
\operatorname{tr} A_{\sigma}^{\prime}=\sum_{i}\left\langle A_{\sigma}^{\prime} e_{i}, e_{i}\right\rangle+\sum_{\alpha}\left\langle A_{\sigma}^{\prime} e_{\alpha}, e_{\alpha}\right\rangle
$$

$$
\operatorname{tr} B_{\sigma}^{\prime}=\sum_{i}\left\langle A_{\sigma}^{\prime} e_{i}, e_{i}\right\rangle
$$

we obtain $\operatorname{tr} Q_{\xi}=\operatorname{tr} Q^{A^{\prime}}$. 
From the above proof, expression (2.14) can also be written as

$$
\operatorname{tr} Q^{A^{\prime}}=\sum_{\sigma, i, \alpha}\left[2\left\langle A_{\sigma}^{\prime} e_{i}, e_{\alpha}\right\rangle^{2}-\left\langle A_{\sigma}^{\prime} e_{\alpha}, e_{\alpha}\right\rangle\left\langle A_{\sigma}^{\prime} e_{i}, e_{i}\right\rangle\right]
$$

At a point $x \in M$, we take an orthonormal basis $\left\{\nu_{\lambda}, \eta_{a}\right\}$ of $V_{x}\left(R^{l}, M\right)$ so that $\left\{\nu_{\lambda}\right\}$ and $\left\{\eta_{\mathrm{a}}\right\}$ are bases of $V_{x}(N, M)$ and $V_{x}\left(R^{l}, N\right)$ respectively. From (2.7), (2.8) and (2.15) we obtain

$$
\operatorname{tr} Q^{A^{\prime}}=\operatorname{tr} Q^{A}+\bar{A}(V)
$$

where $\operatorname{tr} Q^{A}$ is given by (2.5) and

$$
\bar{A}(V)=\sum_{\alpha, i, \alpha}\left[2\left\langle\bar{A}_{\alpha} e_{i}, e_{\alpha}\right\rangle^{2}-\left\langle\bar{A}_{\mathrm{\alpha}} e_{\alpha}, e_{\alpha}\right\rangle\left\langle\bar{A}_{\mathrm{\alpha}} e_{i}, e_{i}\right\rangle\right]
$$

Note that $\bar{A}(V) \neq \operatorname{tr} Q^{\bar{A}}$.

THEOREM. Let $\phi: M^{m} \rightarrow N^{n}$ be an isometric immersion of a compact Riemannian manifold $M$ in a submanifold $N$ of $R^{l}$, and $p$ a given integer, $p \in(0, m)$. Suppose that for any $x \in M$ and any $p$-subspace $V$ of $T_{x} M$,

$$
\operatorname{tr} Q^{A}<-\bar{A}(V) \text {. }
$$

Then there is no stable p-current in $M$ and

$$
H_{p}(M, Z)=H_{m-p}(M, Z)=0 .
$$

PRoOF: Let $\theta$ be the set given by (2.13). If $v^{T} \in \theta, v^{T}$ is the gradient $\nabla f$ of the function $f(x)=\langle v, x\rangle$ on $M$. To each $\mathcal{S} \in \mathcal{R}_{p}(M)$ associate a quadratic form $Q_{s}$ on $\theta$ as follows. For $X \in \theta$ let $\phi_{t}$ be the flow generated by $X$ and set

$$
Q_{S}(X)=\left.\frac{d^{2}}{d t^{2}} M\left(\phi_{t^{*}} \mathcal{S}\right)\right|_{t=0} \text {. }
$$

From (1.2) we have

$$
\operatorname{tr} Q_{S}=\sum_{n} n \int_{S_{n}} \operatorname{tr} Q_{\xi_{n}} d \mathcal{H}^{p}(x) .
$$

But from (1.3), Proposition 1 and (2.16), (2.18) implies $\operatorname{tr} Q_{\xi_{n}}<0$ for any $n$. Therefore $\operatorname{tr} Q_{\mathcal{S}}<0$. This implies that there is no stable $p$-current in $M$. By using FedererFleming's theorem, we have

$$
H_{p}(M, Z)=H_{m-p}(M, Z)=0 .
$$

If $N^{n}$ in the above theorem is a totally umbilical submanifold immersed in $R^{l}$, $N^{n}$ is of constant curvature $c \geqslant 0$. In this case $\bar{A}(V)$ given by (2.17) becomes

$$
\bar{A}(V)=-p(m-p) c .
$$

Hence we obtain 
CoRollary 1. Let $M^{m}$ be a compact submanifold immersed in a totally umbilical submanifold $N^{n}$ of $R^{l}$. If for any $x \in M$ and any $p$-subspace $V$ of $T_{x} M$,

$$
\operatorname{tr} Q^{A}<p(m-p) c
$$

where $c$ is the sectional curvature of $N$, then there is no stable p-current in $M$ and

$$
H_{p}(M, Z)=H_{m-p}(M, Z)=0 \text {. }
$$

REMARK 1. In the case $N^{n}=S^{n}$ we have $c=1$, and Corollary 1 becomes Lawson and Simons' theorem. And when $N^{n}=R^{n}$, Corollary 1 is due to Xin [4, Theorem 1].

\section{Main Results}

Let $m_{1}+m_{2}=m$ and

$$
M^{m}=S^{m_{1}} \times S^{m_{2}}=\left\{\left(x_{1}, x_{2}\right) \in R^{m+2} ; x_{\lambda} \in R^{m_{\lambda}+1} \text { and }\left\|x_{\lambda}\right\|=1, \lambda=1,2\right\} .
$$

Then $M^{m}$ is a submanifold of $R^{m+2}$. At $x=\left(x_{1}, x_{2}\right) \in M^{m}$ we take an orthonormal basis $\left\{\nu_{\lambda}\right\}$ of $V_{x}\left(R^{m+2}, M\right)$ as follows

$$
\nu_{1}=\left(x_{1}, 0\right), \quad \nu_{2}=\left(0, x_{2}\right) .
$$

It may be seen that the shape operators $A_{\lambda}$ can be denoted by the matrices

$$
A_{1}=-\left(\begin{array}{cc}
I_{1} & 0 \\
0 & 0
\end{array}\right), \quad A_{2}=-\left(\begin{array}{cc}
0 & 0 \\
0 & I_{2}
\end{array}\right),
$$

where $I_{\lambda}$ is the $m_{\lambda} \times m_{\lambda}$ identity matrix for each $\lambda=1,2$. Hence for any $X \in T_{x} M$ we have $A_{\lambda} X=-X_{\lambda}$, where $X_{\lambda}$ is the orthogonal projection of $X$ onto $T_{x_{\lambda}} S^{m_{\lambda}}$.

At $x \in M$, we take an orthonormal basis $\left\{e_{i}, e_{\alpha}\right\}$ of $T_{x} M$ so that $\left\{e_{i}\right\}$ is an orthonormal basis of the $p$-subspace $V$. Denoting the orthogonal projection of $e_{i}$ (respectively $e_{\alpha}$ ) onto $T_{x_{\lambda}} S^{m_{\lambda}}$ by $e_{i \lambda}$ (respectively $e_{\alpha \lambda}$ ), we have

$$
\begin{gathered}
\left\langle A_{\lambda} e_{i}, e_{\alpha}\right\rangle=-\left\langle e_{i \lambda}, e_{\alpha \lambda}\right\rangle, \\
\operatorname{tr} A_{\lambda}=\sum_{i}\left\langle A_{\lambda} e_{i}, e_{i}\right\rangle+\sum_{\alpha}\left\langle A_{\lambda} e_{\alpha}, e_{\alpha}\right\rangle=-\sum_{i}\left\|e_{i \lambda}\right\|^{2}-\sum_{\alpha}\left\|e_{\alpha \lambda}\right\|^{2}, \\
\operatorname{tr} B_{\lambda}=\sum_{i}\left\langle B_{\lambda} e_{i}, e_{i}\right\rangle=\sum_{i}\left\langle A_{\lambda} e_{i}, e_{i}\right\rangle=-\sum_{i}\left\|e_{i \lambda}\right\|^{2} .
\end{gathered}
$$

Substituting these into (2.5) we obtain

$$
\begin{aligned}
\operatorname{tr} Q^{A}= & \sum_{i, \alpha}\left[2\left(\left\langle e_{i 1}, e_{\alpha 1}\right\rangle^{2}+\left\langle e_{i 2}, e_{\alpha 2}\right\rangle^{2}\right)\right. \\
& \left.-\left(\left\|e_{\alpha 1}\right\|^{2}\left\|e_{i 1}\right\|^{2}+\left\|e_{\alpha 2}\right\|^{2}\left\|e_{i 2}\right\|^{2}\right)\right] .
\end{aligned}
$$


Since $e_{i}=e_{i 1}+e_{i 2}$ and $e_{\alpha}=e_{\alpha 1}+e_{\alpha 2}$, we have

$$
\begin{gathered}
\left\|e_{i 1}\right\|^{2}+\left\|e_{i 2}\right\|^{2}=1, \quad\left\|e_{\alpha 1}\right\|^{2}+\left\|e_{\alpha 2}\right\|^{2}=1 \\
\left\langle e_{i 1}, e_{\alpha 1}\right\rangle+\left\langle e_{i 2}, e_{\alpha 2}\right\rangle=0
\end{gathered}
$$

So (3.1) becomes

$$
\begin{aligned}
\operatorname{tr} Q^{A}= & \sum_{i, \alpha}\left[4\left\langle e_{i 1}, e_{\alpha 1}\right\rangle^{2}+\left\|e_{i 1}\right\|^{2}+\left\|e_{\alpha 1}\right\|^{2}\right. \\
& \left.-2\left\|e_{i 1}\right\|^{2}\left\|e_{\alpha 1}\right\|^{2}\right]-p(m-p)
\end{aligned}
$$

LEMмA . For each pair of fixed indices $i, \alpha$, let

$$
f_{i \alpha}=4\left\langle e_{i 1}, e_{\alpha 1}\right\rangle^{2}+\left\|e_{i 1}\right\|^{2}+\left\|e_{\alpha 1}\right\|^{2}-2\left\|e_{i 1}\right\|^{2}\left\|e_{\alpha 1}\right\|^{2} .
$$

Then $f_{i \alpha} \leqslant 1$ and equality holds if and only if $e_{i} \in T_{x_{1}} S^{m_{1}}$ and $e_{\alpha} \in T_{x_{2}} S^{m_{2}}$, or $e_{\alpha} \in T_{x_{1}} S^{m_{1}}$ and $e_{i} \in T_{x_{2}} S^{m_{2}}$.

Proof: Let $e_{i}^{*}\left(s=1,2, \ldots, m_{1}\right)$ (respectively $e_{\alpha}^{*}$ ) be the components of $e_{i 1}$ (respectively $\left.e_{\alpha_{1}}\right)$ with respect to an orthonormal basis of $T_{x_{1}} S^{m_{1}}$. Then (3.3) becomes

$$
\begin{aligned}
f_{i \alpha}= & 4\left(\sum_{\bullet} e_{i}^{s} e_{\alpha}^{s}\right)^{2}+\sum_{\bullet}\left(e_{i}^{s}\right)^{2} \\
& +\sum_{*}\left(e_{\alpha}^{s}\right)^{2}-2 \sum_{s, t}\left(e_{i}^{s}\right)^{2}\left(e_{\alpha}^{t}\right)^{2}
\end{aligned}
$$

where

$$
0 \leqslant \sum_{,}\left(e_{i}^{z}\right)^{2} \leqslant 1, \quad 0 \leqslant \sum_{e}\left(e_{\alpha}^{z}\right)^{2} \leqslant 1 .
$$

In order to seek the maximum of $f_{i \alpha}$ under the condition (3.5), partially differentiating (3.4) with respect to each variable and equating to zero, we obtain

$$
\begin{aligned}
& 4\left(\sum_{t} e_{i}^{t} e_{\alpha}^{t}\right) e_{\alpha}^{t}+e_{i}^{t}-2 \sum_{t}\left(e_{\alpha}^{t}\right)^{2} e_{i}^{t}=0, \\
& 4\left(\sum_{t} e_{i}^{t} e_{\alpha}^{t}\right) e_{i}^{t}+e_{\alpha}^{t}-2 \sum_{t}\left(e_{i}^{t}\right)^{2} e_{\alpha}^{t}=0 .
\end{aligned}
$$

These equations can be expressed by

$$
\begin{aligned}
& u e_{i}^{s}=4 w e_{\alpha}^{\prime}, \\
& v e_{\alpha}^{\prime}=4 w e_{i}^{s},
\end{aligned}
$$




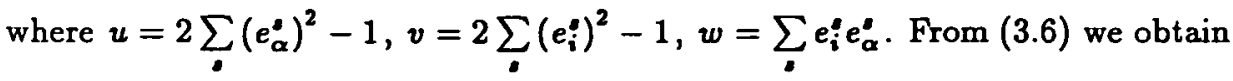

$$
\begin{aligned}
& \frac{1}{2}(1+v) u=4 w^{2}, \\
& u w=2(\check{\gamma}+u) w .
\end{aligned}
$$

Similarly, from (3.7) we have

$$
\begin{aligned}
& \frac{1}{2}(1+u) v=4 w^{2}, \\
& v w=2(1+v) w .
\end{aligned}
$$

(3.8) and (3.10) give $u=v$. If $w \neq 0$, from (3.9) we have $u=2(1+u)$. So $u=-2$ and thus $\sum_{c}\left(e_{\alpha}^{*}\right)^{2}=-1 / 2$; this is impossible. Therefore $w$ must be zero. And from (3.8) we have $(1+v) u=0$, that is,

(i) $1+v=0$; this gives $e_{i}^{t}=0$, and $e_{\alpha}^{s}=0$ from $u=v$; or

(ii) $u=v=w=0$; this implies $\sum_{\alpha}\left(e_{i}^{s}\right)^{2}=\sum_{0}\left(e_{\alpha}^{d}\right)^{2}=1 / 2$ and $\sum_{i} e_{i}^{a} e_{\alpha}^{s}=0$.

From (3.4),

$$
\text { (i) implies } f_{i \alpha}=0 \text {, and (ii) implies } f_{i \alpha}=1 / 2 \text {. }
$$

Besides, if $\sum_{i}\left(e_{i}^{q}\right)^{2}=1$, that is, $\left\|e_{i 1}\right\|^{2}=1$, then $e_{i 2}=0$ and thus

$$
\sum_{j} e_{i}^{a} e_{\alpha}^{\prime}=\left\langle e_{i 1}, e_{\alpha 1}\right\rangle=-\left\langle e_{i 2}, e_{\alpha 2}\right\rangle=0
$$

From (3.4) we have

$$
f_{i \alpha}=1-\sum_{\alpha}\left(e_{\alpha}^{\prime}\right)^{2} \leqslant 1
$$

equality holds if and only if $\sum_{\alpha}\left(e_{\alpha}^{s}\right)^{2}=0$. Combining $\left({ }^{*}\right)$, we see that under the condition (3.5), $f_{i \alpha} \leqslant 1$. Clearly equality holds if and only if $e_{i} \in T_{x_{1}} S^{m_{1}}$ and $e_{\alpha} \in T_{x_{2}} S^{m_{2}}$, or $e_{\alpha} \in T_{x_{1}} S^{m_{1}}$ and $e_{i} \in T_{x_{2}} S^{m_{2}}$.

From this lemma, (3.2) gives

$$
\operatorname{tr} Q^{A}=\sum_{i, \alpha} f_{i \alpha}-p(m-p) \leqslant 0 .
$$

It is easy to check that equality holds if and only if $\left\{e_{i}\right\} \subset T_{x_{1}} S^{m_{1}}$ and $\left\{e_{\alpha}\right\} \subset T_{x_{2}} S^{m_{2}}$, or $\left\{e_{\alpha}\right\} \subset T_{x_{1}} S^{m_{1}}$ and $\left\{e_{i}\right\} \subset T_{x_{2}} S^{m_{2}}$. These imply the p-subspace $V=T_{x_{1}} S^{m_{1}}$ and $V^{\perp}=T_{x_{2}} S^{m_{2}}$, or $V=T_{x_{2}} S^{m_{2}}$ and $V^{\perp}=T_{x_{1}} S^{m_{1}}$. Hence we have 
Proposition 2. For the isometric immersion $S^{m_{1}} \times S^{m_{2}} \rightarrow R^{m+2}$ $\left(m=m_{1}+m_{2}\right), \operatorname{tr} Q^{A} \leqslant 0$. Furthermore, if $p \bar{\in}\left\{m_{1}, m_{2}\right\}, \operatorname{tr} Q^{A}<0$.

Proof of Theorem 1: In the Theorem of Section 2 we take $M^{m}=S^{m_{1}} \times S^{m_{2}}$ and $N^{n}=R^{m+2}$; then $\bar{A}(V)=0$ from (2.17). Combining Proposition 2 and the Theorem in Section 2, we obtain Theorem 1.

Proof of Theorem 2: Let $\left\{e_{i}, e_{\alpha}\right\}$ be an orthonormal basis of $T_{x} M$ so that $\left\{e_{i}\right\}$ is a basis of the $p$-subspace $V$. Note that the shape operators of $S^{n_{1}} \times S^{n_{2}} \rightarrow$ $R^{n_{1}+n_{2}+2}$ are $\bar{A}_{a}(a=1,2), \bar{A}_{a} X=-X_{a}$ where $X \in T_{x} M$ and $X_{a}$ is the orthogonal projection of $X$ onto $T_{x_{a}} S^{n_{a}}$. So $\left\langle\bar{A}_{a} e_{i}, e_{\alpha}\right\rangle=-\left\langle e_{i a}, e_{\alpha a}\right\rangle,\left\langle\bar{A}_{a} e_{i}, e_{i}\right\rangle=-\left\|e_{i a}\right\|^{2}$, and $\left\langle\bar{A}_{a} e_{\alpha}, e_{\alpha}\right\rangle=-\left\|e_{\alpha a}\right\|^{2}$. Thus (2.17) becomes

$$
\bar{A}(V)=\sum_{i, \alpha}\left[2\left(\left\langle e_{i 1}, e_{\alpha 1}\right\rangle^{2}+\left\langle e_{i 2}, e_{\alpha 2}\right\rangle^{2}\right)-\left(\left\|e_{\alpha 1}\right\|^{2}\left\|e_{i 1}\right\|^{2}+\left\|e_{\alpha 2}\right\|^{2}\left\|e_{i 2}\right\|^{2}\right)\right] \text {. }
$$

So from the Lemma we have $\bar{A}(V)=\sum_{i, \alpha} f_{i \alpha}-p(m-p) \leqslant 0$. Combining this with the Theorem in Section 2 we obtain Theorem 2.

CoRollary 2. Let $M^{m}$ be a compact submanifold isometrically immersed in $S^{n_{1}} \times S^{n_{2}}$. If for any point $x \in M$ and any $p$-subspace $V$ of $T_{x} M(0<p<m)$ the selfadjoint linear operator $Q^{A}$ on $V$ is negative definite, then there is no stable $p$ current in $M$.

REMARK 2. Theorems and corollaries in this paper are true if one replaces the integers by any finitely generated abelian coefficient group because the Federer-Fleming theorem remains true in the latter case. Besides, one can easily generalise these theorems and corollaries to arbitrary varifolds on $M$ from [3, p.436, Remark 4].

\section{REFERENCES}

[1] B.-Y. Chen, Geometry of submanifolds (Dekker, New York, 1973).

[2] H. Federer and W. Fleming, 'Normal and integral currents', Ann. of Math. 72 (1960), 458-520.

[3] H.B. Lawson and J. Simons, 'On stable currents and their application to global problems in real and complex geometry', Ann. of Math. 98 (1973), 427-450.

[4] Y.-L. Xin, 'An application of integral currents to the vanishing theorems', Sci. Sinica Ser. $A 27$ (1984), 233-241.

Department of Mathematics

Xian Institute of Metallurgy and

Construction Engineering

Xian 710055

Peoples Republic of China 